\title{
ESTUDIO DE LOS COMPONENTES DE PRODUCCIÓN DE CAFÉ (Coffea arábica L.) VARIEDAD CASTILLO® EN NARIÑO
}

\author{
Study of coffee (Coffea arabica L.) Castillo ${ }^{\circledR}$ variety production \\ components in Nariño
}

Johanna Alixa Muñoz-Belalcazar ${ }^{1}$

E-mail: mjohannaalixa@gmail.com*

Tulio César Lagos-Burbano ${ }^{3}$

E-mail: tclagosb@udenar.edu.co

\section{Carlos Andrés Benavides-Cardona ${ }^{2^{*}}$}

E-mail: cabenavides@udenar.edu.co

Jorge Fernando Navia Estrada ${ }^{4}$

E-mail: jornavia@yahoo.com

${ }^{1}$ Ing. Agrónoma, M.Sc. Universidad de Nariño, sede VIPRI, Facultad de Ciencias Agrícolas, Grupo de Investigación en Producción de Frutales Andinos. San Juan de Pasto - Nariño, Colombia. ${ }^{2}$ Ing. Agrónomo, M.Sc. Universidad de Nariño, sede VIPRI, Facultad de Ciencias Agrícolas, Grupo de Investigación en Producción de Frutales Andinos. San Juan de Pasto - Nariño, Colombia. ${ }^{3}$ Ing. Agrónomo, Ph.D. Universidad de Nariño, sede VIPRI, Facultad de Ciencias Agrícolas, Grupo de Investigación en Producción de Frutales Andinos. San Juan de Pasto - Nariño, Colombia.

${ }^{4}$ Ing. Agrónomo, Ph.D. Universidad de Nariño, sede Torobajo, Facultad de Ciencias Agrícolas.

Fecha recepción: 25 de mayo de 2021 / Fecha Aprobación: 28 de junio 2021 / Fecha Publicación: 30 de julio 2021

\section{RESUMEN}

El presente estudio tuvo por objetivo evaluar la relación de las variables climáticas y de crecimiento, en la producción del cultivo de café Variedad Castillo ${ }$, en el departamento de Nariño. Se seleccionaron tres lotes (1ha), en tres diferentes rangos altitudinales por municipio $(\leq 1600 \mathrm{~m}$, entre $1601 \mathrm{y} 1800 \mathrm{~m}$, $>1800 \mathrm{~m}$ ). Las variables registradas fueron precipitación (PP), temperatura (TEMP), radiación fotosintéticamente activa (RFA), altura de planta (AP), diámetro basal del tallo (DBT), número de hojas $(\mathrm{NH})$, número de ramas primarias (NRP), número de ramas secundarias (NRS), área foliar (AF), índice de área foliar (IAF), número de nudos por rama (NNR) y longitud de ramas (LR). Estas fueron contrastadas con la producción en g.pl-1 de café pergamino seco (CPS/PL). Se realizó un análisis descriptivo de las variables climáticas; un análisis de varianza para variables de crecimiento y producción; un análisis de correlación de Pearson entre las variables evaluadas y un análisis de sendero de las variables que presentaron un mayor coeficiente de correlación. El registro de la RFA fue variable, con respecto a las altitudes y los municipios evaluados. El análisis de varianza mostró diferencias estadísticas, las cuales evidenciaron el efecto de la altitud sobre los parámetros registrados. El análisis de correlación mostró relaciones positivas entre altitud y variables y del rendimiento, con AP, DT, NRP, LR y IAF. Por otra parte, con el análisis de sendero se identificó a las variables AP, NRP y LR, como las de mayores efectos sobre el CPS/PL, en forma transversal, mientras tanto, las variables IAF, NH y NRP, presentaron los mayores efectos directos.

Palabras clave.

Análisis de correlación, análisis de sendero, índice de área foliar, producción.

\section{Cómo citar:}

Muñoz-Belalcazar, J.A.; Benavides-Cardona, C. A.; Lagos-Burbano, T.C y Navia Estrada, J.F. (2021). Estudio de los componentes de producción de café (Coffea arábica L.) variedad castillo® en Nariño. Revista Facultad de Ciencias Agropecuarias -FAGROPEC. Universidad de la Amazonia. Vol. 13 (2), 114-128. https://doi.org/10.47847/fagropec.v13n2a3 


\section{ABSTRACT}

The objective of this study was to evaluate the relationship between climatic and growth variables and the production of Castillo ${ }^{\circledR}$ variety coffee in the department of Nariño. Three plots (1ha-1) were selected in three different altitudinal ranges per municipality $(\leq 1600 \mathrm{~m}$, between 1601 and $1800 \mathrm{~m}$, $>1800 \mathrm{~m}$ ). The variables recorded were precipitation (PP), temperature (TEMP), photosynthetically active radiation (PAR), plant height (PH), basal stem diameter (BD), number of leaves (NL), number of primary branches (NPB), number of secondary branches (NSB), leaf area (LA), leaf area index (LAI), number of nodes per branch (NNB) and branch length (BL), which were compared with the production in g.pl-1 of dry parchment coffee (CPS/PL). A descriptive analysis of the climatic variables, analysis of variance for growth and production variables, Pearson correlation analysis between the variables evaluated and path analysis of the variables with the highest correlation coefficient were carried out. The PAR was variable with respect to the altitudes and municipalities evaluated. The analysis of variance showed statistical differences that evidenced the effect of altitude on the parameters recorded. The correlation analysis showed positive relationships between altitude and variables, and of yield with AP, DT, NRP, LR and IAF, on the other hand, the path analysis identified the variables AP, NRP and LR, as those with the greatest effects on the CPS/PL in a transversal way; while the variables IAF, NH and NRP presented the greatest direct effects.

\section{Key words.}

Correlation analysis, path analysis, leaf area index, production.

\section{INTRODUCCIÓN}

En la actualidad, el café es parte integral de la economía de más de 70 países (Moncada et al., 2016), proporcionando un medio de vida para más de 25 millones de agricultores (Panhuysen $\&$ Pierrot, 2014) (McNougher, 2013). Su consumo a nivel mundial se encuentra en continuo crecimiento; en tal sentido, de 57,9 millones de sacos que se consumían en 1964, se proyecta que habrá un consumo de 166,3 millones de sacos, para el año cafetero 2020/21. A su vez, se estima que ésta tendencia se mantendrá, si se tiene en cuenta el incremento del consumo en países como China, Corea del sur y Rusia, los cuales tradicionalmente no son consumidores (OIC, 2021; OIC, 2016). Por su parte, la producción del grano ha tendido a estabilizarse o reducirse, debido principalmente a factores como los fenómenos del Niño y de La Niña (Sauchyn et al., 2016); también debido a las características del suelo y al manejo del cultivo; a la aparición de plagas; al sistema de labranza; a la densidad de plantas y de la topografía, entre otros factores (Lince \& Sadeghian, 2015) (FNC, 2015) (Suárez et al., 2014). Del mismo modo, la falta de implementación y de adopción de tecnología es causada por los factores mencionados y la por la poca uniformidad en la cosecha (Ocampo \& Álvarez, 2017) (Salazar et al., 2016) (Araujo e Silva Ferras et al., 2012).

La tendencia actual en la producción de los cultivos se encamina hacia una producción sostenible y amigable con el ambiente, que permita garantizar una mayor competitividad en los mercados internacionales (Confalone et al., 2006); en este sentido, el conocimiento sobre la conformación fisiológica de la producción es de gran importancia, en tanto permite optimizar 
el manejo de aquellas variables más influyentes en la productividad del cultivo (Ramírez et al., 2010). Para lograr lo anterior, se requiere de estudios detallados, in situ, que permitan analizar las causas de la variación de la producción y su relación con sus componentes. Siguiendo esta línea, el presente estudio se planteó con el objeto de evaluar la relación de las variables climáticas y de crecimiento, con la producción del cultivo de café Variedad Castillo®, en cuatro municipios cafeteros del departamento de Nariño.

\section{MATERIALES Y MÉTODOS}

\section{Localización}

El estudio se llevó a cabo durante el periodo agrícola que comprende, de agosto de 2016 hasta agosto 2017. Este se implementó en los municipios de La Unión (ecotopo cafetero 220A), cuya ubicación geográfica de referencia se encuentra entre los $1^{\circ} 21^{\prime}$ y $1^{\circ} 42^{\prime \prime} \mathrm{LN}$ y hace parte de las cuencas del Rio Patía y Rio Mayo, Sandoná, Consacá y La Florida (ecotopo cafetero 221A), ubicados entre $1^{\circ} 05^{\prime}$ y $1^{\circ} 36^{\prime}$ " LN y que forman parte de la Cuenca del Río Guaitara (Gómez et al., 1991).

\section{Área experimental}

En cada municipio se seleccionaron tres localidades experimentales, en tres diferentes rangos altitudinales, definidos para el estudio, los cuales son los siguientes: bajo ( $<1600$ m.s.n.m), medio (entre 1600 y 1800 m.s.n.m) y alto (>1800 m.s.n.m). Cada localidad contó con un área de 1ha, establecidas con café Variedad Castillo® de tres años y con una densidad de siembra de 6800pl.ha ${ }^{-1}(1,3 \times 1,3 \mathrm{~m}$ al triángulo), en un sistema a libre exposición solar. En estas se consideraron parámetros homogéneos de manejo agronómico, durante el tiempo de evaluación (Tabla 1).

\section{Variables evaluadas}

A partir del estudio se realizó seguimiento a variables climáticas, de crecimiento y de producción. Dentro de las primeras se consideró la temperatura ambiente (TMP), la precipitación (PP) y la radiación fotosintéticamente activa (RFA), mediciones que a su vez fueron registradas durante el periodo de evaluación, por estaciones meteorológicas de Spectrum Technologies inc., modelo 2900ET, Weather Station, ubicadas en cada lote experimental.

Para la evaluación de las variables de crecimiento, en cada localidad (1ha), se tomaron al azar dieciséis (16) plantas, en las cuales, cada sesenta (60) días, durante el periodo de evaluación, se midieron las siguientes variables: altura de planta en cm (AP), desde la base del suelo hasta el último ápice formado; diámetro basal de tallo en $\mathrm{cm}$ (DBT), estimado a $10 \mathrm{~cm}$ de la superficie del suelo; número de hojas $(\mathrm{NH})$, número de ramas primarias $(\mathrm{NRP})$, secundarias $(\mathrm{NRS})$ y 


\section{Tabla 1.}

Ubicación geográfica de las localidades experimentales de la investigación.

\begin{tabular}{clccc}
\hline Municipio & Localidad & Latitud (W) & Longitud (N) & Altitud (M) \\
\hline \multirow{2}{*}{ Sandoná } & Las Delicias & $1^{\circ} 13^{\prime} 36^{\prime \prime}$ & $77^{\circ} 44^{\prime} 54^{\prime \prime}$ & $1536 \mathrm{~B}$ \\
& Mana 1 & $1^{\circ} 10^{\prime} 25^{\prime \prime}$ & $77^{\circ} 48^{\prime} 32^{\prime \prime}$ & $1700 \mathrm{M}$ \\
& La Cruz & $1^{\circ} 12^{\prime} 27^{\prime \prime}$ & $77^{\circ} 46^{\prime} 45^{\prime \prime}$ & $2015 \mathrm{~A}$ \\
Consacá & Cariaco Bajo & $1^{\circ} 10^{\prime} 30^{\prime \prime}$ & $77^{\circ} 28^{\prime} 07^{\prime \prime}$ & $1577 \mathrm{~B}$ \\
& Bomboná & $1^{\circ} 11^{\prime} 26^{\prime \prime}$ & $77^{\circ} 27^{\prime} 31^{\prime \prime}$ & $1668 \mathrm{M}$ \\
& San Antonio & $1^{\circ} 12^{\prime} 50^{\prime \prime}$ & $77^{\circ} 25^{\prime} 56^{\prime \prime}$ & $1989 \mathrm{~A}$ \\
La Florida & $1^{\circ} 22^{\prime} 06^{\prime \prime}$ & $77^{\circ} 17^{\prime} 56^{\prime \prime}$ & $1600 \mathrm{~B}$ \\
& La Joya & $1^{\circ} 23^{\prime} 56^{\prime \prime}$ & $77^{\circ} 18^{\prime} 53^{\prime \prime}$ & $1800 \mathrm{M}$ \\
& Santa Ana & $1^{\circ} 22^{\prime} 20^{\prime \prime}$ & $77^{\circ} 20^{\prime} 43^{\prime \prime}$ & $2030 \mathrm{~A}$ \\
& S. Francisco & $1^{\circ} 38^{\prime} 28^{\prime \prime}$ & $77^{\circ} 09^{\prime} 00^{\prime \prime}$ & $1430 \mathrm{~B}$ \\
& La Playa & $1^{\circ} 34^{\prime} 23^{\prime \prime}$ & $77^{\circ} 07^{\prime} 38^{\prime \prime}$ & $1620 \mathrm{M}$ \\
& El Sauce & $1^{\circ} 34^{\prime} 23^{\prime \prime}$ & $77^{\circ} 07^{\prime} 38^{\prime \prime}$ & $2030 \mathrm{~A}$ \\
\hline
\end{tabular}

número de nudos por rama (NNR), estimados por conteo manual; longitud de ramas primarias en cm (LR), con el promedio de 6 ramas de las partes baja, media y alta de la planta; área foliar por planta en $\mathrm{cm}^{2}(\mathrm{AF})$, calculada a través del siguiente modelo matemático:

$$
A F=a+b x / 1+c x+d x 2
$$

Dónde: $\mathrm{a}=4,541, \mathrm{~b}=2,38, \mathrm{c}=0,154 \mathrm{~d}=0,0075 \mathrm{y} \mathrm{x}=$ longitud promedio de seis hojas (parte baja, media y alta de la planta).

Posteriormente, el AF se multiplicó por el número total de hojas por planta, para obtener el área foliar por planta y, finalmente, para estimar el índice de área foliar (IAF), para el cual se dividió el AF por planta, entre el área del suelo que ocupa la planta $(A I F=A F / A S)$.

La variable dependiente producción correspondió a la cosecha del periodo evaluado 20162017, expresado en g.pl ${ }^{-1}$ de café pergamino seco (CPS/PL), para su estimación. Previo a la cosecha se seleccionaron, al azar, sesenta (60) plantas por hectárea en cada parcela experimental; se contó y pesó el número total de frutos por planta; luego se tomaron, al azar, cuatro (4) muestras de cien frutos por localidad, para obtener el peso promedio y para estimar así las producciones por planta y por hectárea.

La relación café cereza versus café pergamino seco - CPS se determinó, a partir de diferenciales de peso. En cada localidad se tomaron muestras de $5 \mathrm{~kg}$ de café cereza, las cuales fueron sometidas a un proceso de beneficio tradicional; se secaron a libre exposición solar y se registró su peso, cuando el grano alcanzo un promedio de $12 \%$ de humedad, con el fin de estimar las relaciones de producción de CPS por planta y por hectárea. 


\section{Análisis de la información}

Para cada localidad se realizó un análisis descriptivo de las variables climáticas registradas, considerando los rangos altitudinales, para las variables de crecimiento y de producción se realizó un ANOVA, con la utilización de la prueba de Tukey, con nivel de significancia de $\mathrm{p}<0,05$, para aquellas variables que evidenciaron diferencias estadísticas. Posteriormente, se realizó un análisis de correlación de Pearson para todas las variables, en cada localidad, y se incluyeron los parámetros de altitud, temperatura promedio y precipitación acumulada, con el objetivo de establecer la asociación entre las variables y la producción del cultivo, permitiendo demostrar la magnitud y la dirección de los efectos de factores en la determinación de caracteres complejos (Valencia \& Ligarreto, 2012).

Las variables que mostraron mayor correlación con la producción se sometieron a un análisis de Sendero, utilizando el software InfoStat, versión 2008 (Balzarini et al., 2008), para evaluar la importancia de cada uno de los componentes en la conformación de la producción y para determinar las relaciones directas e indirectas entre sus características morfológicas o de una variable sobre otra, de acuerdo con los sugerido por Behrouz y DeEll (2009) y por Jasso de Rodríguez et al. (2001).

\section{RESULTADOS Y DISCUSIÓN}

El comportamiento de la temperatura promedio mensual no varió en forma considerable, entre altitudes en cada municipio o entre ellos. Independientemente de la ubicación de las localidades, la temperatura promedio mensual estuvo en un rango, para las altitudes bajas, de entre 19,08 a $20,87^{\circ} \mathrm{C}$; para las medias, de entre 18,49 a $19,94^{\circ} \mathrm{C}$ y, para las altas, de entre 17,14 a $18,35^{\circ} \mathrm{C}$. La temperatura promedio más alta se dio en la altitud baja del municipio de Sandoná, la cual, a pesar de no ser la zona más baja de evaluación, presentó un mayor valor con $23^{\circ} \mathrm{C}$. Por otra parte, en las localidades con altitudes altas de los municipios de Consacá y La Unión se presentó el promedio más bajo de temperatura, con $17^{\circ} \mathrm{C}$ en ambos casos. La FNC (2010) indicó que la temperatura óptima para el crecimiento y para el desarrollo del cultivo estaba entre 17 y $23^{\circ} \mathrm{C}$, valores que, para las zonas de estudio, estuvieron dentro del rango independientemente de su altitud.

La precipitación evidenció un comportamiento uniforme en cuanto a su distribución, independiente de la altitud o del municipio evaluado; los picos más altos se observaron en los periodos correspondientes al último trimestre de 2016 y al segundo trimestre de 2017. El Municipio con mayor precipitación fue La Unión, con 1493,5, 1896,14 y 2733,17 mm, en las altitudes baja media y alta respectivamente; la menor precipitación se presentó en La Florida, con $1308,06,796,0, y 1605,77 \mathrm{~mm}$ en las altitudes baja, media y alta respectivamente.

Ramírez et al. (2013) afirman que la precipitación óptima para el cultivo de café se encuentra entre 1600 y $1800 \mathrm{~mm}$ anuales y, aunque esta es la indicada, es posible producir con niveles o 
frecuencia de precipitación diferentes, situación que se evidenció en las localidades evaluadas. La reducción o abundancia de las lluvias en el cultivo de café, además se traduce en la reducción de su actividad fisiológica, afectando los procesos de crecimiento y de producción (Turbay et al., 2014) (Avelino \& Rivas, 2013) (Laderach et al., 2011). Sin embargo, en el momento de la diferenciación floral se requiere de un déficit hídrico, que garantice alta floración. Además, la demanda hídrica del cultivo depende de la edad, de las características del suelo y de la altitud (Ramírez et al., 2010).

Por otra parte, el comportamiento de la radiación fotosintéticamente activa-RFA fue variable, con respecto a las altitudes y municipios evaluados, con valores que oscilaron, desde los $472,3 \mu \mathrm{mol} \cdot \mathrm{m}^{-2} \cdot \mathrm{s}^{-2}$, obtenidos en la altitud alta de La Unión, hasta los 975,75 $\mu \mathrm{mol} \cdot \mathrm{m}^{-2} \cdot \mathrm{s}^{-2}$, observados en la altitud baja de La Florida; además, en este municipio se presentaron los mayores promedios de la variable, en las tres altitudes evaluadas $(975,75,853,3$ y $802 \mu \mathrm{mol} . \mathrm{m}$ ${ }^{2} \cdot \mathrm{s}^{-2}$, alta, media y baja respectivamente). Este factor se consideró de gran importancia, ya que su su influencia se tradujo en crecimiento vegetal, producción de biomasa y rendimiento (Rojas, 2015; Castilla et al., 2013). Un aumento de este parámetro induce la formación de plantas más bajas, así como mayor diferenciación y, por ende, que estas sean más productivas; mientras tanto, un estadio bajo, sombrío o con menores valores de RFA, estimularía la formación de plantas más altas, con menor diferenciación y menos productivas (Arcila et al., 2007).

\section{Crecimiento y producción}

La prueba de comparación de medias de Tukey (Tabla 2), para las variables que evidenciaron diferencias estadísticas, en el análisis de varianza realizado con el fin de observar el efecto de la altitud, sobre los parámetros considerados, mostré que, para el municipio de La Florida, el comportamiento de las variables que se diferenciaron estadísticamente, con respecto al factor altitud (Baja, Media y Alta), fueron los siguientes: DBT (3,29, 2,91,3,64cm, respectivamente); $\mathrm{NH}\left(733,04,576,39,942,21\right.$, en su orden); AF $\left(105233,96,81544,74,135838,76 \mathrm{~cm}^{2}\right)$ y IAF $(7,21,5,59,9,3)$. La altitud baja difirió de la media y de la alta, superándolas en valores, para las variables NRP $(55,38,44,53)$ y CPS/PL $\left(806,94,142,59\right.$ y $\left.309,9 \mathrm{~g} \cdot \mathrm{pl}^{-1}\right)$.

En el municipio de La Unión, las variables que presentaron diferencias estadísticas, según el rango altitudinal, Bajo, Medio y Alto, fueron AP (139,75, 144,94, $125 \mathrm{~cm}$, en su orden); DBT $(3,21,3,47,3,78 \mathrm{~cm}$, respectivamente); $\mathrm{NH}(487,12,666,22,608,1)$; $\mathrm{AF}(65927,89,90240,83$, $\left.8646,67 \mathrm{~cm}^{2}\right)$ y IAF $(4,52,6,18,5,92)$. La altitud media presentó similitud estadística con la altitud alta, registrando los mayores valores para las variables NH, AF y IAF. En Sandoná se presentaron diferencias estadísticas, según la zona baja, media y alta, con respecto a las variables $\mathrm{NH}(602,84,615,31,699,99$ en su orden $)$, AF $\left(86928,41,88952,31,100530,95 \mathrm{~cm}^{2}\right)$ y $\operatorname{IAF}(5,96,6,09,6,89)$; la altitud alta difirió estadísticamente con la altitud media y baja, en las variables NH, AF y IAF. En el municipio de Consacá se encontraron diferencias estadísticas significativas, para las altitudes baja, media y alta, con respecto a la variable CPS/PL (69,2, 
Tabla 2.

Comparación de medias para las variables DBT, NH, NRP, AF, IAF, CPS/PL y AP, en parcelas de café variedad castillo, en tres rangos altitudinales, de cuatro municipios de la zona cafetera de Nariño.

\begin{tabular}{|c|c|c|c|c|c|c|c|c|c|}
\hline Municipio & & & & & Iriables & & & & \\
\hline \multirow[t]{4}{*}{ La Florida } & Zona & DBT & NH & & NRP & & $\mathbf{A F}$ & IAF & CPS/PL \\
\hline & Baja & $3,29 \mathrm{~B}$ & 733,04 & $\mathrm{~B}$ & 55,38 & $\mathrm{~B}$ & $105233,96 \mathrm{~B}$ & $7,21 \quad \mathrm{~B}$ & $806,94 \mathrm{~B}$ \\
\hline & Media & $2,91 \mathrm{~A}$ & 576,39 & A & 44 & A & $81544,74 \mathrm{~A}$ & $5,59 \mathrm{~A}$ & $142,59 \mathrm{~A}$ \\
\hline & Alta & $3,64 \mathrm{C}$ & 942,21 & $\mathrm{C}$ & 53 & $\mathrm{AB}$ & $135838,76 \mathrm{C}$ & $9,30 \quad \mathrm{C}$ & $309,40 \mathrm{~A}$ \\
\hline \multirow[t]{4}{*}{ La Unión } & Zona & $\mathbf{A P}$ & DBT & & NH & & $\mathbf{A F}$ & IAF & \\
\hline & $\overline{\text { Baja }}$ & $139,75 \mathrm{AB}$ & 3,21 & $\mathrm{~A}$ & 487,12 & $\mathrm{~A}$ & $65927,89 \mathrm{~A}$ & $4,52 \mathrm{~A}$ & \\
\hline & Media & $144,94 \mathrm{~B}$ & 3,47 & A B & 666,22 & $\mathrm{~B}$ & $90240,83 \mathrm{~B}$ & $6,18 \quad \mathrm{~B}$ & \\
\hline & Alta & $125 \mathrm{~A}$ & 3,78 & $\mathrm{~B}$ & 608,10 & $\mathrm{~B}$ & $86467,67 \mathrm{~B}$ & $5,92 \quad \mathrm{~B}$ & \\
\hline \multirow[t]{4}{*}{ Sandoná } & Zona & NH & $\mathbf{A F}$ & & IAF & & & & \\
\hline & Baja & $602,84 \mathrm{~A}$ & 86928,41 & $\mathrm{~A}$ & 5,95 & $\mathrm{~A}$ & & & \\
\hline & Media & $615,31 \mathrm{~A}$ & 88952,31 & A & 6,09 & A & & & \\
\hline & Alta & $699,99 \mathrm{~B}$ & 100530,95 & $\mathrm{~B}$ & 6,89 & $\mathrm{~B}$ & & & \\
\hline \multirow[t]{4}{*}{ Consacá } & Zona & CPS/PL & & & & & & & \\
\hline & Baja & $69,20 \mathrm{~A}$ & & & & & & & \\
\hline & Media & $225,50 \mathrm{~B}$ & & & & & & & \\
\hline & Alta & $112,10 \mathrm{AB}$ & & & & & & & \\
\hline
\end{tabular}

Nota: medias con una letra común no son significativamente diferentes $(\mathrm{p}>0,05)$.

225,5, 112,1gc.p.s.pl ${ }^{-1}$ en orden), que difirió entre las altitudes baja y media, presentando el mayor valor, pero sin diferenciarse con la zona de altitud alta.

El AF y el IAF, al igual que el NH, presentaron diferencias estadísticas con respecto a las altitudes en los municipios de Sandoná, La Florida y La Unión; según Montoya et al. (2017), la relación entre la producción de café cereza y el área foliar estimada del árbol, indica que por cada $100 \mathrm{~cm}^{2}$ de $\mathrm{AF}$, la producción del árbol se incrementa en $2,37 \mathrm{~g}$ de café cereza; del mismo modo, como lo afirma Silva et al. (2011), a mayor área foliar, generalmente hay mayor capacidad de interceptación de radiación solar y, por ende, mayor productividad.

El mayor IAF para este estudio se ubicó en la altitud alta de la Florida, con 9,3, razón por la cual, posiblemente, esta zona fue una de las que evidenció diferencias, con respecto a la producción de CPS/PL. Este es un indicador del uso eficiente del recurso suelo, de la captación de energía y de la productividad. De acuerdo con Bermúdez (2016), el IAF óptimo para Coffea arabica L., variedad Caturra, es de 8; en investigaciones realizadas por Alvarado y Ochoa (2006), en Variedad Castillo, se registró un IAF de 7 y 8, a los tres y a los cuatro años respectivamente, con una densidad de siembra de 10.000 plantasha $^{-1}$. 
El NRP mostró diferencias estadísticas únicamente en La Florida, en donde la altitud baja obtuvo el mayor número $(55,38)$; esto podría explicar el comportamiento de las variables antes mencionadas (NH, AF, IAF). Durante el ciclo de producción anual, para el desarrollo de los frutos, una planta de café requiere hasta cuatro veces más fotoasimilados, que los que se necesita para la generación de una nueva rama, y se espera que, con mayor número de ramas, haya una mayor producción; lo anterior, a su vez depende de factores climáticos como la radiación solar (Vaast et al., 2005) (Balladares \& Calero, 2005).

Así entonces, de forma general se pudo observar que el comportamiento asociado con el crecimiento del cultivo, si bien, en algunas localidades presentó diferencias, con respecto a las variaciones altitudinales y a las variables evaluadas, esto no representa un comportamiento predecible y diferencial definitivo, para municipios en forma particular. Es por esto necesario un análisis, no solo de las relaciones existentes entre variables para la producción, sino también, del grado de aporte que estas hacen a la misma.

Con respecto a las variaciones que fueron encontradas podemos afirmar que, en muchos de los paramentos identificados, fue posible encontrar coincidencias con aportes realizados por diversos autores, con respecto al comportamiento de los mismos; por ejemplo, desde el Centro Nacional de Investigaciones de Café - Cenicafé (2011), se afirma que las variaciones de temperatura influyen directamente sobre la longitud de entrenudos, de altura de la planta y de floración, aspecto que también se puede evidenciar en el presente trabajo, ya que al no identificarse un rango de variación amplio, en la temperatura promedio, a través de los municipios y de las altitudes evaluadas, únicamente se pudieron evidenciar diferencias estadísticas, en un solo municipio (La Unión), para la variable AP; incluso en este aspecto, esa diferencia podría asumirse con respecto a la variable climática RFA, la que, precisamente en esta zona, reflejó valores variables que pudieron incidir, como lo indica Arcila et al. (2007), considerando la AP como una variable que aporta al desarrollo de ramas primarias y secundarias, que garanticen la producción (Balladares \& Calero, 2005).

Por su parte, el DBT y NH considerados por Bustamante et al (2004), como ítems de importancia, especialmente a partir del segundo año de la producción en un cultivo de café, para este trabajo se establecieron como las variables que más difirieron estadísticamente, con respecto a las altitudes en La Florida y en La Unión para DBT y en Sandoná, La Florida y La Unión para NH.

\section{Análisis de correlación}

El análisis de correlación para el municipio de Sandoná evidenció, en gran medida, el comportamiento descrito anteriormente, con respecto a las variables de crecimiento versus las variaciones altitudinales de la zona, tales como AP vs NRP $(0,54)$; las variables AF e IAF vs ALT, TEMP y PP presentaron 0,99 en todos los casos; NNR vs LR mostraron 0,89 y, finalmente, considerando como variable dependiente producción CPS/PL, esta mostró que las 
variables RFA $(0,57)$ y DBT $(0,66)$ fueron las que más se asociaron con este parámetro.

Para el municipio de La Unión, la ALT se mostró como la variable correlacionada, en mayor medida, con RFA, PP y TEMP (-0,99 en los tres casos); con AP (-0,65); con DBT $(0,75)$; con NRS $(0,58)$ y con AF e IAF con $(0,59$ en ambos casos). Con respecto a la producción CPS/PL, las variables que más se correlacionaron fueron $\mathrm{AP}(0,57), \mathrm{NH}(0,61), \mathrm{AF}$ y IAF $(0,54)$; variables, que como se mencionó anteriormente, se consideraron como las responsables de garantizar una mayor zona de floración y una mayor fructificación.

Los valores de correlación más evidentes, en el municipio de Consacá, se observaron en las variables ALT, con RFA $(0,9)$ y PP $(1,0)$; con TEMP $(0,99)$; AP con NH $(0,82)$, LR $(0,88), \mathrm{NH}$ con AF e IAF $(0,99$ en ambos casos). Además, se pudo evidenciar que las variables $\mathrm{NH}(0,62)$, $\operatorname{AF}$ e IAF $(0,66)$ fueron las que presentaron una mayor correlación, con el rendimiento CPS/PL.

Finalmente, en el municipio de La Florida la ALT conservó su valor de correlación con respecto a RFA $(-0,98)$ y TEMP $(-0,99)$; RFA presentó alta correlación con TEMP $(0,98)$. Para las variables de crecimiento AP se encontró alta correlación con $\operatorname{NRP}(0,72), \operatorname{AF}$ e IAF $(0,67$ en los dos casos); DBT con NH, AF e IAF (0,9 en los tres casos); NH con PP, AF e IAF (0,99 en las tres variables). Además, se pudo evidenciar que las variables $\operatorname{ALT}(-0,7)$, RFA $(0,78)$ y NRS $(0,62)$ fueron las que presentaron una mayor correlación con CPS/PL y que las variables AF, $\operatorname{IAF}(0,12)$ y NNR $(-0,01)$ fueron las que presentaron los coeficientes más bajos de correlación, con la variable dependiente CPS/PL.

Siguiendo esta línea, vale decir que Valencia y Ligarreto (2012) indican que los coeficientes no muestran la importancia que tienen los efectos directos e indirectos, que estos tienen sobre la variable rendimiento, ya que un coeficiente de correlación alto o bajo, entre dos variables, se puede deber al efecto de una tercera variable o a un grupo de variables.

\section{Análisis de sendero}

El análisis de sendero permitió establecer los efectos directos, de cada una de las variables seleccionadas, sobre la producción del cultivo, así como los efectos entre variables, que afectan igualmente a la productividad (efectos indirectos), en las cuatro localidades evaluadas (Tabla 3).

Por su parte, en el municipio de Sandoná las variables RFA $(0,57)$ y DBT $(0,66)$ mostraron los mayores efectos totales significativos, sobre la producción de café Variedad Castillo. Las variables RFA $(0,73)$, NRS $(-0,52)$ y AP $(0,41)$ presentaron los mayores efectos directos. Alvarado y Ochoa (2006) afirman que la altura de planta es una de las variables que guarda una estrecha relación, con el número de ramas y con la producción. Al incrementarse la altura de la planta aumenta el número de nudos y de ramas $\mathrm{y}$, por ende, también la productividad; lo 
Tabla 3.

Contribución directa e indirecta de las variables ALT, RFA, AP, DBT, NH, NRP, NRS, AF, IAF, NNR, LR, PP y TEMP, con respecto a la variable dependiente rendimiento (CPS/PL), analizadas sobre el rendimiento de plantas de café variedad Castillo, en cuatros municipios del departamento de Nariño.

\begin{tabular}{|c|c|c|c|c|c|c|c|}
\hline Municipio & Variable & RFA & AP & DBT & NRP & NRS & Corr.con CPS/PL \\
\hline \multirow{6}{*}{ Sandoná } & RFA & 0,73 & $-0,04$ & 0,17 & $-0,06$ & $-0,23$ & 0,57 \\
\hline & $\mathbf{A P}$ & $-0,07$ & 0,41 & 0,09 & 0,15 & $-0,31$ & 0,26 \\
\hline & DBT & 0,28 & 0,08 & 0,44 & 0,1 & $-0,25$ & 0,66 \\
\hline & NRP & $-0,16$ & 0,22 & 0,17 & 0,27 & $-0,18$ & 0,31 \\
\hline & NRS & 0,32 & 0,24 & 0,21 & 0,1 & $-0,52$ & 0,36 \\
\hline & & AP & NH & NRP & IAF & LR & Corr, con CPS/PL \\
\hline \multirow{5}{*}{ La Unión } & $\mathbf{A P}$ & 0,69 & $-0,03$ & $-0,03$ & $-0,03$ & $-0,03$ & 0,57 \\
\hline & NH & 0,07 & $-0,27$ & $-0,01$ & 0,83 & $-0,01$ & 0,61 \\
\hline & NRP & 0,41 & $-0,07$ & $-0,05$ & 0,13 & $-0,04$ & 0,37 \\
\hline & IAF & $-0,02$ & $-0,26$ & $-0,01$ & 0,84 & $-0,03$ & 0,59 \\
\hline & LR & 0,37 & $-0,04$ & $-0,04$ & 0,07 & $-0,06$ & 0,3 \\
\hline \multirow{6}{*}{ Consacá } & & RFA & NH & NRP & IAF & LR & Corr, Con CPS/PL \\
\hline & $\begin{array}{c}\text { RFA } \\
\text { NH }\end{array}$ & $\begin{array}{c}\mathbf{- 3 , 7 6} \\
0,31\end{array}$ & $\begin{array}{c}3,52 \\
\mathbf{- 4 2 , 6 7}\end{array}$ & $\begin{array}{l}0,74 \\
2,26\end{array}$ & $\begin{array}{l}-0,14 \\
40,61\end{array}$ & $\begin{array}{l}0,03 \\
0,12\end{array}$ & $\begin{array}{l}0,36 \\
0,62\end{array}$ \\
\hline & NRP & $-1,01$ & $-35,2$ & 2,73 & 33,89 & 0,11 & 0,52 \\
\hline & IAF & 0,01 & $-42,5$ & 2,27 & 40,77 & 0,12 & 0,66 \\
\hline & LR & $-0,04$ & $-31,01$ & 1,8 & 29,48 & 0,16 & 0,39 \\
\hline & & ALT & RFA & NRP & NRS & TEMP & Corr, con CPS/PL \\
\hline \multirow{5}{*}{ La Florida } & ALT & 5,99 & $-6,84$ & 0,22 & $-0,33$ & 0,26 & $-0,7$ \\
\hline & RFA & $-5,92$ & 6,92 & $-0,33$ & 0,37 & $-0,26$ & 0,78 \\
\hline & NRP & $-1,33$ & 2,36 & $-0,97$ & 0,5 & $-0,06$ & 0,5 \\
\hline & NRS & $-3,33$ & 4,3 & $-0,81$ & 0,6 & $-0,15$ & 0,62 \\
\hline & TEMP & $-5,99$ & 6,82 & $-0,2$ & 0,33 & $-0,26$ & 0,69 \\
\hline
\end{tabular}

anterior, entendiendo que el número de ramas productivas es un índice de mayor producción (Balladares \& Calero, 2005). A su vez, la variable (AP) mostró un efecto indirecto sobre el rendimiento, a través del número de ramas secundarias (-0,31). El NRP presentó un efecto indirecto sobre el rendimiento, a través de la $\operatorname{RFA}(0,32)$ y AP $(0,24)$.

Con respecto al municipio de La Unión, las variables AP $(0,57)$, NH $(0,61)$ e IAF $(0,54)$ mostraron los mayores efectos totales significativos, sobre la variable dependiente CPS/PL; las variables AP $(0,69)$ e IAF $(0,84)$ presentaron los mayores efectos directos. En tal sentido, una mayor área foliar total implica, generalmente, una mayor capacidad de intercepción de 
radiación solar y, por ende, una mayor productividad; por lo anterior, se considera importante tener conocimiento sobre el comportamiento de esta variable de crecimiento (Silva et al., 2011). De igual modo, el NH mostró un efecto indirecto significativo sobre el rendimiento, a través de IAF $(0,83)$; NRP presentó un efecto indirecto significativo sobre el rendimiento, a través de altura de planta $(0,41)$.

Ahora bien, con respecto al municipio de Consacá, las variables NH $(0,62)$ e IAF $(0,66)$ presentaron los mayores efectos totales significativos, sobre la variable dependiente CPS/PL; por su parte, las variables NH $(-42,67)$ e IAF $(40,77)$ presentaron los mayores efectos directos. El NH mostró un efecto indirecto significativo sobre el CPS/PL, a través de IAF $(40,61)$; el NRP presentó un efecto indirecto significativo sobre el CPS/PL, a través del IAF $(33,89)$. Como lo cita Balladares y Calero (2005), el aumento del número de ramas es una característica morfológica del café, relacionada con la productividad y, como lo menciona Silva et al. (2011), una mayor área foliar total implica, generalmente, una mayor capacidad de intercepción de radiación solar y, por ende, una mayor productividad.

Finalmente, en el municipio de La Florida las variables ALT $(-0,7)$, RFA $(0,78)$ y TEMP $(0,69)$ presentaron los mayores efectos totales significativos, sobre la variable dependiente CPS/PL; las variables ALT $(5,99)$ y RFA $(6,92)$ presentaron los mayores efectos directos. El NRP mostró un efecto indirecto significativo sobre el CPS/PL, a través de la RFA (2,36); a su vez, la TEMP presentó un efecto indirecto significativo sobre el CPS/PL, a través de la RFA $(683,2)$.

\section{CONCLUSIONES}

Durante la evaluación, el comportamiento climático se mostró variable, con respecto a la precipitación acumulada, la cual, no evidenció diferencias apreciables, en relación con su distribución y con picos máximos de ocurrencia. A su vez, para las altitudes y las zonas evaluadas, e independientemente de las condiciones de evaluación, los rangos climáticos no presentaron oscilaciones significativas.

Por su parte, la altitud fue un parámetro que guardó estrecha relación con las variables de crecimiento evaluadas, en especial, en el municipio de La Unión. Los parámetros de crecimiento de mayor correlación con el rendimiento fueron altura de planta, número de hojas, longitud de ramas, área foliar e índice de área foliar en forma transversal, para los cuatro municipios evaluados.

Finalmente, las variables que mayor aporte significativo presentaron, como parámetros de crecimiento, fueron la altura de planta, el número de hojas, el número de ramas principales y la longitud de las ramas. Como parámetros climáticos, la radiación fotosintéticamente activa, la altitud y la temperatura, tuvieron efectos sobre el rendimiento, únicamente en la localidad de la Florida, razón por la cual, no se puede afirmar que el comportamiento productivo fue transversal, para las demás localidades del mismo ecotopo, es decir para Sandoná y Consacá. 


\section{AGRADECIMIENTOS}

Los autores del presente estudio agradecen al Grupo de Investigación en Producción de Frutales Andinos de la Universidad de Nariño y al proyecto de investigación denominado: "Investigación evaluación del efecto de sombra de diferentes especies arbóreas en el comportamiento agronómico y calidad de Café, Consacá, Nariño, Occidente", el cual es financiado por el SGR.

\section{REFERENCIAS}

Alvarado, G., Ochoa, H. (2006). Características fenotípicas de componentes de variedad Castillo en dos ambientes. Cenicafé, 57(2), 100-121. https://www,cenicafe,org/es/publications/arc057\%2802\%29100-121.pdf

Araujo e Silva Ferraz, G., Da Silva, F., De Carvalho, M. (2012). Análisis geoestadístico de rendimiento de frutos y fuerza de desprendimiento en café. Precisión Agric 13, 76-89. https://link,springer,com/article/10,1007/s11119-011-9223-8

Arcila, J., Farfan, F., Moreno, A., Salazar, L., Hincapie, E. (2007), Sistemas de producción de café en Colombia, Chinchiná, Cenicafé, 309p. https://biblioteca,cenicafe,org/bitstream/10778/720/1/Sistemas $\% 20$ producci $\% \mathrm{C} 3 \% \mathrm{~B} 3 \mathrm{n} \%$ 20caf $\%$ C3\%A9\%20Colombia.pdf

Avelino, J., Rivas, G. (2013). La roya anaranjada del cafeto. https://hal,archivesouvertes, fr/hal-01071036

Balladares, D., Calero, J. (2005). Efecto de la sombra y fertilización sobre el crecimiento, estructura productiva y calidad de café (Coffea arabica) vr, Costa Rica 95. [Tesis de grado, Universidad Nacional Agraria] https://repositorio,una,edu,ni/1969/1/tnf04b188,pdf

Balzarini, M., Gonzalez, L., Tablada, M., Casanoves, F., Di Rienzo, J., Robledo, C. (2008), InfoStat Software Estadístico. Manual del Usuario. Editorial Brujas.

https://www,researchgate,net/publication/283569520 Software estadistico_InfoStat_Ma nual de usuario Version_1/link/5f5f8745a6fdcc116410c707/download

Behrouz, M., De Ell, J. (2009). Correlation and path-coefficient analyses of ripening attributes and storage disorders in 'Ambrosia' and 'Empire' apples. Revista Postharvest Biology and Technology, 51 (2), 168-173. https://doi,org/10,1016/j,postharvbio,2008,07,006

Bermúdez, L. (2016). Evaluación del crecimiento y producción en diferentes condiciones 
de siembra en café variedad Castillo [Tesis de Maestría, Universidad Nacional de Colombia, Medellín Colombia].

Bustamante, J., Casanova, A., Roman, N., Monterey, C. (2004). Estimación temprana del potencial de rendimiento en café (Coffea arabica L,) Var, Bramón I. Bioagro, 16(1), 3-8. http://www,ucla,edu,ve/bioagro/Rev16(1)/1,\%20Estimaci\%C3\%B3n\%20temprana.pdf

Castilla, N., Baeza, E., Papadopoulos, A. (2013). Greenhouse technology and management (2a ed). CABI Publishing, .

Centro Nacional de Investigaciones de Café - CENICAFE. (2011). Variedad climática y la floración del café en Colombia. CENICAFE. https://www,cenicafe,org/es/publications/avt04072,pdf

Confalone, M., Castro, B., Ruiz, B., Sau, F. (2006). Componentes del rendimiento en leguminosas de grano con posibilidades de ser utilizadas como forraje invernal en Galicia. Pastos, 36 (2), 177-192.

Federación Nacional de Cafeteros de Colombia -FNC. (2015). Comportamiento de la industria cafetera colombiana 2014. FNC.

Federación Nacional de Cafeteros de Colombia- FNC. (2010). Centro nacional de investigaciones de café - Cenicafé, Chinchiná, Colombia. FNC.

International Coffee Organization - OIC. (2021). Coffee prices rallied in April 2021 amid an anticipated decline in production and signs of economic recovery from covid-19 pandemic. OIC. https://www,ico,org/documents/cy2020-21/cmr-0421-e.pdf

Jasso de Rodríguez, D., Angulo, L., Rodríguez, G. (2001). Correlation and path coefficient analyses of the agronomic trait of a native population of guayule plants. Revisit Industrial Crops and Products, 14(2), 93-103. https://doi,org/10,1016/S0926-6690(00)00092-3

Laderach, P., Lundy, M.,Jarvis, A., Ramirez, J., Perez, E., Schepp, K. (2011). Predicted Impact of Climate Change on Coffee Suppy Chains. (Conference paper) DOI: 10,1007/978-3-642-14776-0_42

Lince, L., Sadeghian, S. (2015). Producción de café (Coffea arabica L,) en función de las propiedades del suelo, en dos localidades de Quindío, Colombia. Revista de Investigación Agraria y Ambiental,7 (1), 71. https://hemeroteca,unad,edu,co/index,php/riaa/article/view/1547/1844

McNougher, P. (2013). Insight special: Debunking coffee myths, Coffee division of ED\&F. 
MAN. https://www,yumpu,com/en/document/read/22891202/insight-special-debunkingcoffee-myths-bijdendijk

Moncada, M., Tovar, E., Montoya, J., González, A., Spindel, J., McCouch, S. (2016). A genetic linkage map of coffee (Coffea arabica L,) and QTL for yield, plant height, and bean size. Tree Genetics \& Genomes 12 (5),1-17.

Montoya, R., Hernández, A., Unigarro, M., Flórez, R. (2017). Estimación del área foliar en café variedad Castillo a libre exposición y su relación con la producción. Revista Cenicafé 68(19), 55-61

Ocampo, O., Álvarez, L, (2017), Tendencia de la producción y el consumo del café en Colombia. Apuntes del CENES, 36(64), 139-165. DOI:

https://doi,org/10,19053/01203053,v36,n64,2017,5419

Organización Internacional del Café -OIC. (2016). Informe del mercado de café- febrero 2016, Aumenta el consumo mundial de café, pero los precios siguen bajos, Londres. OIC. http://www,scielo,org,co/pdf/cenes/v36n64/0120-3053-cenes-36-64-00139,pdf

Panhuysen, S., Pierrot, J. (2014). Barómetro de café 2014. Ensayos sobre economía cafetera, 27 (30).

Ramos, L., Criollo, H. (2017). Calidad física y sensorial de Coffea arábica L, variedad Colombia, perfil Nespresso AAA, Unión Nariño. Rev, Cienc, Agr, 34(2), 83 - 97. DOI: https://doi,org/10,22267/rcia, 173402,74

Ramírez, V., Arcila, J., Jaramillo, A., Rendón, J., Cuesta, G., Menza, H., Mejía, C., Montoya, D., Mejía, J., Torres, J., Sánchez, P., Baute, J., Peña, A. (2010). Floración del café en Colombia y su relación con la disponibilidad hídrica, térmica y de brillo solar, Cenicafé, 61(2), 132-158.

Ramírez, V., Jaramillo, A., Arcila, J. (2013). Factores climáticos que intervienen en la producción de café en Colombia, En Federación Nacional de Cafeteros de Colombia FNC y Centro de Investigaciones en café - CENICAFÉ. Manual de cafetero colombiano, Investigación y tecnología para la sostenibilidad de la caficultura. FNC - CENICAFÉ.

Rojas, E. (2015). La radiación PAR y su efecto en los índices de crecimiento, rendimiento y calidad del cultivo de tomate, Centro de Investigación en Química Aplicada [Tesis de Maestría]. https://ciqa,repositorioinstitucional,mx/jspui/bitstream/1025/49/1/TESIS\%20ELVA\%20L ILIANA\%20ROJAS\%20SANCHEZ\%20final,pdf 
Salazar, K., Arroyave, A., Ovalle, A., Ocampo, O., Ramírez, C., Oliveros, C. (2016). Tiempos en la recolección manual tradicional de café. Ingeniería Industrial, 37(2), $114-126$.

Sauchyn, D., Upegui, J., Masiokas, M., Ocampo, O., Cara, L., Villalba, R. (2016). Exposure of Rural Communities to Climate Variability and Change: Case Studies from Argentina, Colombia and Canada. EnW, Leal Filjo, K, Adamson, R, M, Dunk, U, Alzeiteiro, S, Illingworth \& F, Alves (Eds,), Implementing Climate Change Adaptation in Cities and Communities, Integrating Strategies and Educational Approaches (pp, 23-38). Springer. http://doi,org/10,1007/978-3-319-28591-7

Silva, W., Brinate, S., Tomaz, M., Amaral, J., Rodriguez, W., Martíns, L. (2011). Methods to estimate leaf área in coffee. Enciclopedia Biosfera, 7(7), 46-75.

Suarez, J., Rodríguez, E., Duran, E. (2014). Efecto de las condiciones de cultivo, las características químicas de suelo y el manejo de grano en los atributos sensoriales de café (Coffea arabica L, ) en taza. Acta Agron 64(4), 342-348, doi:

http://dx, doi,org/10,15446/acag,v64n4,44641

Turbay, S., Nates, B., Jaramillo, F.,Vélez, J., Ocampo, O. (2014). Adaptación a la variabilidad climática entre los caficultores de las cuencas de los ríos Porce y Chinchiná, Colombia, Investigaciones Geográficas. Boletín del Instituto de Geografía, 0(85), 95-112. http://doi, org/10,14350/rig,42298

Vaast, P., Angrand, J., Franck, N., Dauzat, J., Génard, M. (2005). Fruit load and branch ring-barking affect carbon allocation and photosynthesis of leaf and fruit of Coffea arabica in the field. Tree Physiology, 25, 753-760, https://doi,org/10,1093/treephys/25,6,753

Valencia, R., Ligarreto G. (2012), Correlación fenotípica y análisis de sendero para el rendimiento de soya (Glycine max (L) Merril). Revista Acta Agronómica, 61 (4). https://revistas, unal,edu,co/index,php/acta_agronomica/rt/printerFriendly/38137/40313 